\title{
Acknowledgement to Referees for Skin Appendage Disorders 2018
}

Skin

Appendage Disorders

The Editors extend their gratitude and appreciation to the following reviewers whose comments and criticisms ensure the quality of the papers published in this journal.

Roberto Arenas, Tlalpan, Mexico Daniel Asz Sigall, Mexico City, Mexico Robert Baran, Cannes, France Richert Bertrand, Brussels, Belgium Vincenzo Bettoli, Ferrara, Italy Ulrike Blume-Peytavi, Berlin, Germany Pierre Bouhanna, Paris, France Anna Campanati, Ancona, Italy Marie Caucanas, Toulouse, France Ralph Daniel, Jackson, USA James Q. Del Rosso, Henderson, USA Nilton Di Chiacchio, São Paulo, Brazil Ncoza Dlova, Durban, South Africa Isabella Doche, São Paulo, Brazil Judith Dominguez-Cherit, Mexico City, Mexico

Aline Donati, São Paulo, Brazil Boni Elewski, Birmingham, USA Laila El Shabrawi-Caelen, Graz, Austria Gabriella Fabbrocini, Naples, Italy Harold Farber, Philadelphia, USA Steven Feldman, Winston-Salem, USA Maria Fernanda Gavazzoni-Dias, Niterói, Brazil

Lynne Goldberg, Boston, USA

Stamatios Gregoriou, Athens, Greece Ramon Grimalt, Sant Cugat del Vallès, Spain

Chander Grover, Delhi, India

Aditya K. Gupta, London, Canada

Daniela Guzman-Sanchez, Guadalajara, Mexico

Eckart Haneke, Freiburg, Germany Maria K. Hordinsky, Minneapolis, USA Matilde Iorizzo, Bellinzona, Switzerland Nathaniel Jellinek, East Greenwich, USA Gregor B.E. Jemec, Roskilde, Denmark Ana Kaminsky, Buenos Aires, Argentina Jean Kanitakis, Lyon, France Alexandros Katoulis, Athens, Greece Yanna Kelly, São Paulo, Brazil Brett Andrew King, New Haven, USA Francesco Lacarrubba, Catania, Italy Clifford Lawrence, UK Pauline Lecerf, Brussels, Belgium
Dong-Youn Lee, Seoul, Republic of Korea Monika Franziska Linek, Hamburg, Germany María Abril Martinez-Velasco, Mexico City, Mexico Amy J. McMichael, Winston-Salem, USA Andrew G. Messenger, Sheffield, UK Giuseppe Micali, Catania, Italy Paradi Mirmirani, Vallejo, USA Mariya Miteva, Miami, USA Bianca Maria Piraccini, Bologna, Italy Rodrigo Pirmez, Rio de Janeiro, Brazil Adriana Rakowska, Warsaw, Poland Efstathios Rallis, Athens, Greece Alfredo Rebora, Genoa, Italy Pascal Reygagne, Paris, France Phoebe Rich, Portland, USA Bertrand Richert, Brussels, Belgium Evan A. Rieder, New York, USA Nicole E. Rogers, Metairie, USA Ricardo Romiti, São Paulo, Brazil Adam I. Rubin, Philadelphia, USA Lidia Rudnicka, Warsaw, Poland Cecilia Sandoval Tress, Puerto Vallarta, Mexico

Richard K. Scher, New York, USA Jerry Shapiro, New York, USA Avner Shemer, Tel Hashomer, Israel Robert Silverman, Washington, USA Rodney Sinclair, East Melbourne, Australia Sidharth Sonthalia, Gurugram, India Michela Starace, Bologna, Italy Catherine Stefanato, London, UK Ralph M. Trüeb, Wallisellen, Switzerland Sergio Vañó-Galván, Madrid, Spain Norma Vazquez, Monterrey, Mexico Colombina Vincenzi, Bologna, Italy Tracey C. Vlahovic, Philadelphia, USA Annika Vogt, Berlin, Germany Ronni Wolf, Rehovot, Israel Uwe Wollina, Dresden, Germany Martin Zaiac, Miami Beach, USA Christos C. Zouboulis, Dessau, Germany 